\title{
Aspects of Molecular Chirality at Metal Surfaces
}

\author{
By Karl-Heinz Ernst*
}

Laboratory for Nanoscale Materials Science, Empa, Swiss Federal Laboratories for Materials Testing and Research, Überlandstrasse 125, CH-8600 Dübendorf, Switzerland

Dedicated to Prof. Dr. Klaus Christmann on the occasion of his $65^{\text {th }}$ birthday

(Received August 1, 2008; accepted October 5, 2008)

\section{Chirality / Crystallization / Enantiomorphism / Scanning Tunneling Microscopy}

\begin{abstract}
Phenomena like transfer, expression and amplification of chirality in molecular monolayers are reviewed. Chirality can be bestowed onto achiral surfaces by adsorption of chiral molecules. This offers a good opportunity to study two-dimensional chiral crystallization phenomena, like lateral resolution of enantiomers or the transfer of handedness from single molecules into mesoscopic ensembles at high resolution with scanning probe microscopy. Induction of homochirality on surfaces via cooperatively amplified interactions in molecular monolayers is a new phenomenon of supramolecular surface chirality. Prochiral molecules will turn into either handedness upon adsorption, but doping with intrinsically chiral molecules breaks this symmetry and induces homochirality. A similar effect is induced by a small enantiomeric excess. The excess molecules provide the chiral bias that becomes amplified into single lattice chirality.
\end{abstract}

\section{Introduction}

In his famous experiment in 1848 Pasteur separated left- and right-handed ammonium sodium tartrate crystals manually and observed opposite optical activity for their aqueous solutions [1]. His insight that the origin of chirality is based on molecular structure laid the foundation of modern structural organic chemistry. Two important details of his experiment actually allowed the manual separation: i) Handedness was transferred from the molecular structure to the macroscopic shape of the crystal (hemihedrism), and ii) the racemate precipitated into a conglomerate of homochiral crystals. The underlying mechanisms of both processes are still poorly understood. We are neither able to predict the shape of a

\footnotetext{
* Corresponding author. E-mail: karl-heinz.ernst@empa.ch
} 
crystal from molecular structure [2], nor do we know why less than ten percent of all racemates crystallize into conglomerates [3, 4]. One reason for the difficulty to understand and investigate these processes is their cooperative nature. Extremely small structural influences govern the macroscopic result when they become amplified by many cooperating units. For similar reasons, the transfer of chirality into mesoscopic structures - a very important issue in liquid crystal science - is difficult to predict in three dimensions. Cooperativity among different homochiral biomolecules is also of fundamental importance in life [5].

More traceable and easier understood are two-dimensional (2D) crystals formed at surfaces. In particular, this allows studying these processes at the molecular level by exploitation of scanning tunneling microscopy (STM). Different aspects of chirality in two-dimensional molecular lattices formed on the $\mathrm{C}_{3} \mathrm{~V}$ and $\mathrm{C}_{2} \mathrm{~V}$-symmetric single-crystalline copper surfaces are here reviewed. Reasoning for the choice of $\mathrm{Cu}$ as substrate is the relative mobility of the molecules, allowing intermolecular recognition to come into play. A more noble metal would require cooling to lower temperatures in order to study $2 \mathrm{D}$ crystals with STM, more reactive metals interfere with self-assembly due to low mobility. The examples from our own previously published work include monolayers of enantiopure and racemic heptahelicene $\left(\mathrm{C}_{30} \mathrm{H}_{18}\right)$, a helically shaped aromatic hydrocarbon, on $\mathrm{Cu}(111)$, the buckybowl corannulene $\left(\mathrm{C}_{20} \mathrm{H}_{10}\right)$ on $\mathrm{Cu}(110)$, racemic tartaric acid $\left(\mathrm{C}_{4} \mathrm{H}_{6} \mathrm{O}_{6}\right)$ on $\mathrm{Cu}(110)$ as well as chirally doped meso-tartaric acid and succinic acid $\left(\mathrm{C}_{4} \mathrm{H}_{6} \mathrm{O}_{4}\right)$ on $\mathrm{Cu}(110)$.

\section{Experimental details}

All adsorbate systems have been investigated under ultra-high vacuum (UHV) conditions $\left(p \approx 10^{-8} \mathrm{~Pa}\right.$ ). The organic compounds were evaporated from Knudsen cells in vacuo onto the copper crystal surfaces at room temperature. The polished $\mathrm{Cu}$ crystal surfaces $(\mathrm{MaTecK})$ have been prepared as described in detail previously [6]. Cleanliness and quality of the surfaces and the surface coverage of the adsorbate systems have been determined via X-ray photoelectron spectroscopy (XPS), low energy electron diffraction (LEED), temperature programmed desorption (TPD) and STM. TPD curves have been acquired with a heating rate of $4 \mathrm{~K} \mathrm{~s}^{-1}$ using a quadrupole mass spectrometer as detector. Synthesis and enantiomeric separation (ee $>99.9 \%$ ) of heptahelicene $([7] \mathrm{H})$ has been performed as described previously [7, 8]. The absolute configuration was assigned with a high level of confidence by comparison of experimental and calculated VCD spectra [9]. X-ray photoelectron diffraction studies (XPD) of $(M)$-[7]H on the stepped $\mathrm{Cu}(332)$ surface also confirmed this assignment [10]. Tartaric acid $(R, S-, R, R-$, $S, S$ - \& racemate) and succinic acid were purchased from Aldrich and Merck with purities better than $99 \%$. STM images were acquired in constant-current mode with the sample slowly cooled to $50 \mathrm{~K}$. Molecular modeling calculations 
(MMC) were performed using the AMBER force field of the Hyperchem 7 package.

\section{Results and discussion}

\subsection{Transfer of chirality into two-dimensional molecular layers}

Pure enantiomers at surfaces form in most cases 2D enantiomorphous structures in close-packed monolayers [11]. That is, chirality is transferred from the single molecule into a long-range chiral motif. The created motifs are mirror images for the opposite enantiomers. Examples are the pure enantiomers [7]H on $\mathrm{Cu}(111)$ [12]. Close to monolayer saturation, handed pinwheel structures are observed via STM (Fig. 1a, b) in the lattice ( $\beta$-structure). The $(M)$-[7]H-pinwheels have the opposite handedness than the $(P)$-[7]H-pinwheels. In addition, at full monolayer coverage ( $\gamma$-structure), "three-molecule-cloverleaf" units show opposite tilt angles with respect to the adsorbate lattice unit cell (Fig. 1(c,d)). The adsorbate lattice is aligned in an oblique angle to the underlying substrate lattice. Especially this latter mode of expression of chirality in ordered adsorbate systems is a common phenomenon $[11,13]$. Because STM does not unambiguously allow assignment of the relative orientation of the molecules, we performed molecular modeling calculations (MMC) for the $\gamma$-structure. In particular, results from previous XPD studies for this system were very valuable in order to define reasonable starting conditions. Namely, we know that the terminal phenanthrene group is located parallel to the surface plane, i.e., the helix spirals away from the surface. Moreover, six azimuthal orientations co-exist on $\mathrm{Cu}(111)$ [10]. With this information, we placed four molecules in equal orientations at the corners of a unit cell and allowed the others within the unit cell to adopt different substrate sides and azimuthal orientations. This procedure reveals that this $[7] \mathrm{H}$ structure is governed by steric constraints. The lowest-energy structure for the close-packed monolayer (Fig. 1(c,d)) delivers identical adsorption sites for all molecules of the unit cell. Their respective azimuthal orientations, however, are different and depend strongly on the sign of helicity of the molecules, so that opposite lattice enantiomorphism is observed for the enantiomers. Assuming that the brightest features of the STM images reflect the off-centered topmost part of the molecular helix, the MMC-structure in Fig. 1e agrees well with the experimentally observed STM structure in Fig. 1c [12]. Similar to the frustrated lattice structures observed for crystalline polymers of single helicity, e. g., isotactic poly(propylene) [14], not all helices can be aligned "in phase". This shows that, although the packing is mediated by the $\mathrm{Cu}$ substrate grid, the "one-pitch" [7] $\mathrm{H}$ helices follow the same sterically controlled packing rules as extended helical molecules in 3D crystals. [7] $\mathrm{H}$ on $\mathrm{Cu}(111)$ therefore is an example for shortrange sterically controlled supramolecular self-assembly mediated by the surface lattice. 


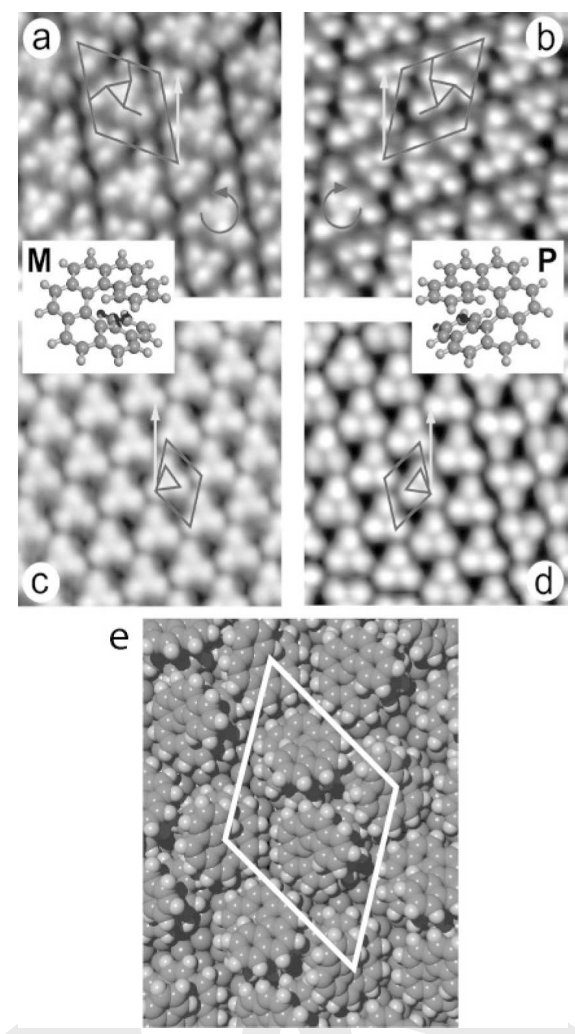

Fig. 1. (a-d) High-resolution STM images $(10 \mathrm{~nm} \times 10 \mathrm{~nm})$ of $(M)$ - and $(P)$-[7]H structures. The respective adlattices have opposite tilt angles with respect to the substrate lattice. (M)-[7]H forms at $91 \%$ of the saturated monolayer clockwise rotated pinwheels, the $\beta(M)$ structure (a), while counterclockwise rotated pinwheels are observed via STM for $(P)-[7 \mathrm{H}]$, the $\beta(P)$ structure (b). For the saturated monolayer lattices $(\gamma(M)$ - (c) and $\gamma(P)$-structure $(d))$, opposite tilt angles of cloverleaf clusters with respect to the adlattice are observed, indicated by triangles in the unit cells, (c,d). (e) Model for the $M$-[7]H cloverleaf structure obtained from MMC. Minimal repulsion is achieved for certain relative azimuthal orientations.

\subsection{Racemic crystals versus lateral separation of enantiomers}

It has been predicted that two-dimensional enantiomeric resolution on a surface should occur more easily than in 3D crystals. Due to confinement in the plane certain symmetry elements, like the center of inversion or the glide plane parallel to the surface, for example, are precluded and enhanced chiral interactions are expected. For lateral resolution of $[7] \mathrm{H}$ on $\mathrm{Cu}(111)$, the same structures as observed for the isolated enantiomers are then expected. Nevertheless, racemic [7]H forms heterochiral lattice structures $[15,16]$. However, mirror domains are observed via LEED and STM [7b, 15]. Molecular recognition, and therefore the 


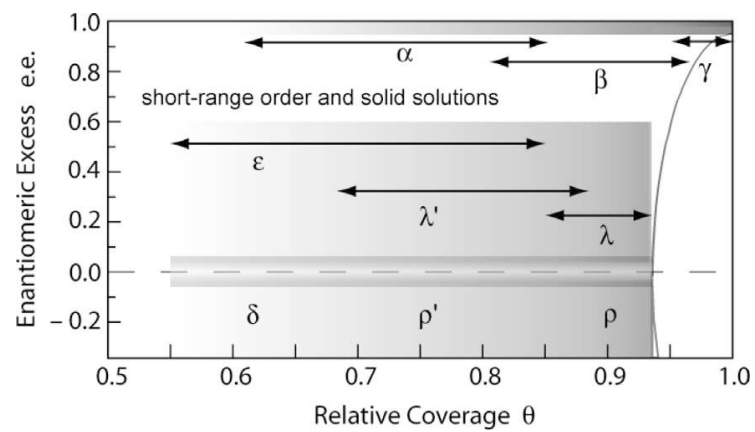

Fig. 2. Phase diagram (coverage vs. ee) of [7]H structures. The coverage ranges, in which the respective structures are observed are indicated by arrows. Since only connected via a mirror operation, the ranges for $\varepsilon, \lambda$, and $\lambda$ apply equally to $\delta, \rho^{\prime}$, and $\rho$, respectively. Not shown are the $\alpha(P), \beta(P)$, and $\gamma(P)$ phases at ee $=-1$.

outcome of lateral enantiomeric separation, is expected to depend on the orientation with respect to the substrate. For $\mathrm{Ni}(100)$, we found a tilted orientation via NEXAFS [17], i. e. no parallel alignment of the hexagonal carbon rings with the surface. We assign this difference to the (100) surface symmetry not allowing the perfect parallel match of the $\mathrm{C} 6$ rings as much as observed for the $\mathrm{Cu}(111)$ surface. On Ni(111), however, a similar match is expected, since the molecule can adopt the small differences. We rather assign the lack of enantioselectivity in self-assembly to the lower mobility of [7] H here, because we did not observe any differences in structures for racemate and pure enantiomers both Ni surfaces [18].

Shown in Fig. 2 is the phase diagram for the different [7]H structures with varying coverage and enantiomeric excess (ee). The diagram is quasi symmetric in ee, so we only plotted the range down to ee $=-0.2$. Overall six pairs of enantiomorphous structures are observed, namely, $\varepsilon / \delta, \lambda^{\prime} / \rho^{\prime}$, and $\lambda / \rho$ for the racemate and $\alpha(P) / \alpha(M), \beta(P) / \beta(M)$, and $\gamma(P) / \gamma(M)$ for the pure enantiomers [16]. Each structure of the racemate exists in two mirror-domains, i.e., $\varepsilon$ is the mirror domain of $\delta$ while $\rho^{\prime}$, and $\rho$ are mirror domains of $\lambda^{\prime}$ and $\lambda$, respectively. Structural details of all observed structures are presented in Table 1.

All adlattices of the racemate have the common feature that the molecules are aligned in zigzag double rows. The lattice structure of the $\lambda / \rho$ phase has been determined via high-resolution STM in combination with extended Hückel simulations as well as MMC (Fig. 3). Its enantiomorphism is based on two possible relative alignments of the two molecules in a heterochiral pair. A glide plane along [110] interconverts the two enantiomers, but is not a symmetry element of a single domain lattice.

The first ordered phase observed with increasing coverage is the $\varepsilon / \delta$ phase (Fig. 2), with molecules aligned at different azimuthal orientations in the double row than observed for the $\lambda / \rho$ phase. Intriguingly, the $\varepsilon / \delta$ phase has a larger 
Table 1. Matrix notation and lattice properties for one set of enantiomorphous [7]H structures on $\mathrm{Cu}(111)$, i. e., enantiopure $\alpha(P), \beta(P), \gamma(P)$, and racemic $\varepsilon, \rho$, and $\rho$ structures. The corresponding mirror structures $\alpha(M), \beta(M), \gamma(M), \lambda^{\prime}, \varepsilon$, and $\lambda$, respectively, are identical in all properties, except matrix notation and sign of tilt angle. The values with error margins are experimental results, the other are expectation values for the lattice defined by the matrix. $a$, $b$ : length of adlattice unit cell vectors; $\alpha$ : angle between $a \& b ; \beta$ : adlattice tilt angle w. r. t. the [110] substrate direction; $A$ : occupied area per molecule; $\theta^{\text {local }}$ : relative coverage of the a perfect structure $(\gamma \equiv 100 \%)$.

\begin{tabular}{|c|c|c|c|c|c|c|c|c|}
\hline lattice & $\begin{array}{c}\text { matrix } \\
\text { notation }\end{array}$ & $\begin{array}{c}\text { molec. } \\
\text { per } \\
\text { unit } \\
\text { cell }\end{array}$ & $a[\AA]$ & $b[\AA]$ & $\alpha_{a, b}$ & $\beta_{[1 \overline{1} 0]}$ & $\begin{array}{c}A / \text { molec } \\
{\left[\AA^{2}\right]}\end{array}$ & $\theta_{\text {rel }}^{\text {local }}$ \\
\hline$\alpha(P)$ & - & 3 & $22.5 \pm 0.5$ & $22.5 \pm 0.5$ & $120^{\circ} \pm 2.5^{\circ}$ & $\approx-22^{\circ} \pm 3^{\circ}$ & 146 & $67 \%$ \\
\hline $\begin{array}{l}\beta(P) \\
(6 \& 3)\end{array}$ & $\left(\begin{array}{cc}12 & -2 \\
2 & 14\end{array}\right)$ & 9 & $\begin{array}{c}33.48 \\
33.3 \pm 0.2\end{array}$ & $\begin{array}{c}33.48 \\
33.3 \pm 0.2\end{array}$ & $\begin{array}{c}120^{\circ} \\
119.9^{\circ} \pm 2.0\end{array}$ & $\begin{array}{c}-7.6^{\circ} \\
-7.7^{\circ} \pm 0.6^{\circ}\end{array}$ & 107.9 & $90.7 \%$ \\
\hline $\begin{array}{l}\gamma(P) \\
\text { (clover) }\end{array}$ & $\left(\begin{array}{cc}8 & 2 \\
-2 & 6\end{array}\right)$ & 3 & $\begin{array}{c}18.41 \\
18.4 \pm 0.2\end{array}$ & $\begin{array}{c}18.41 \\
18.4 \pm 0.2\end{array}$ & $\begin{array}{c}120^{\circ} \\
13.3^{\circ} \pm 2.7^{\circ}\end{array}$ & $\begin{array}{c}+13.89^{\circ} \\
13.3^{\circ} \pm 2.7^{\circ}\end{array}$ & 97.8 & $100 \%$ \\
\hline$\varepsilon$ & $\left(\begin{array}{cc}3 & -2 \\
7 & 8\end{array}\right)$ & 2 & $\begin{array}{c}11.13 \\
11.7 \pm 0.6\end{array}$ & $\begin{array}{c}19.27 \\
19.6 \pm 0.5\end{array}$ & $\begin{array}{c}90^{\circ} \\
90.7^{\circ} \pm 2.6^{\circ}\end{array}$ & $\begin{array}{c}-23.41^{\circ} \\
-22^{\circ} \pm 2.2^{\circ}\end{array}$ & 107.2 & $91.3 \%$ \\
\hline$\rho^{\prime}$ & $\left(\begin{array}{cc}4 & -1 \\
6 & 10\end{array}\right)$ & 2 & $\begin{array}{c}11.69 \\
11.9 \pm 0.3\end{array}$ & $\begin{array}{c}22.25 \\
22.4 \pm 0.3\end{array}$ & $\begin{array}{c}94.31^{\circ} \\
94.8^{\circ} \pm 1.1^{\circ}\end{array}$ & $\begin{array}{c}-10.89^{\circ} \\
-10.5 \pm 0.8^{\circ}\end{array}$ & 129.6 & $75.3 \%$ \\
\hline$\rho$ & $\left(\begin{array}{cc}4 & -1 \\
5 & 8\end{array}\right)$ & 2 & $\begin{array}{c}11.69 \\
11.7 \pm 0.5\end{array}$ & $\begin{array}{c}17.87 \\
18.0 \pm 0.2\end{array}$ & $\begin{array}{c}92.67^{\circ} \\
92.7^{\circ} \pm 1.6^{\circ}\end{array}$ & $\begin{array}{c}-10.89^{\circ} \\
-10.5 \pm 0.8^{\circ}\end{array}$ & 104.4 & $93.7 \%$ \\
\hline
\end{tabular}

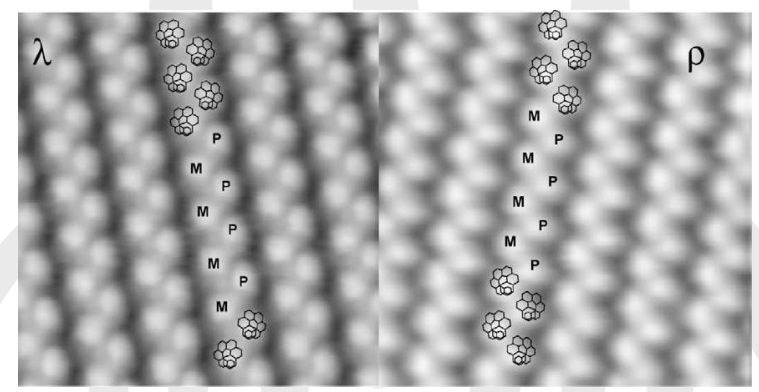

Fig. 3. Racemic [7]H on $\mathrm{Cu}(111)$. Superposition of the molecular orientation and STM images $(10 \mathrm{~nm} \times 10 \mathrm{~nm})$ of the mirror domains $(\lambda \& \rho)$ of the highest coverage monolayer structure. The observed enantiomorphism results from an alignment of heterochiral pairs in opposite chiral conformations.

local density than the $\lambda^{\prime} / \rho^{\prime}$ intermediate phase (Table 1 ), which is observed at higher global coverage (Fig. 2). This leads to the (counterintuitive) situation that with increasing coverage a lower-density-phase forms. Apparently, the azimuthal orientation within the $\varepsilon / \delta$ phase becomes sterically unfavorable with increasing coverage (and therefore lateral pressure). Locally, a less favored structure is formed, but globally the mixture of $\varepsilon / \delta$ and $\lambda^{\prime} / \rho^{\prime}$ present a more stable situation. The abrupt switch in orientation between both phases is explained by the fact 


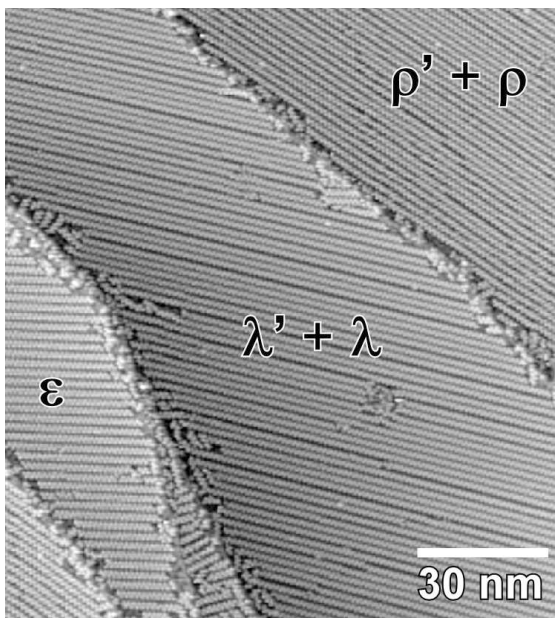

Fig. 4. STM image showing coexistence of an $\varepsilon$ domain and a conglomerate of $\lambda^{\prime} / \lambda$ and $\rho^{\prime} / \rho$ domains. Each domain covers a complete terrace, i.e., domain boundaries coincide with monoatomic steps of the substrate. The $\varepsilon$ structure (and its mirrored $\delta$ structure) is observed upon increasing coverage before appearance of the lower-density $\lambda$ ' structure (see Table 1 ). Conversion from $\lambda^{\prime}$ to $\lambda$ and $\rho$ ' to $\rho$ runs continuously.

that the substrate allows only distinct alignments. Figure 4 shows these structures coexisting at intermediate coverage. The $\lambda^{\prime} / \rho^{\prime}$ phase, in turn, is a precursor of the highest-coverage $\lambda / \rho$ phase. It has identical orientations of the molecules in a double row, but at larger inter-row distances.

The opposite racemate structures only coexist at small ee (Section 3.5). Small amounts of the opposite enantiomer, on the other hand, act as chiral impurities and suppress the long range order, so that the enantiopure structures are only observed at ee close to \pm 1 . Below a coverage of half a monolayer as well as from ee $=|0.5|$ to ee $=|0.95|$ basically no long-range order is present and the two enantiomers form a solid solution. Figure 2 also shows that the pure enantiomers can be packed more tightly than the racemate. Higher density means higher gain in adsorption enthalpy with respect to the molecules in the gas phase, and this should strongly favor formation of enantiopure domains and therefore lateral separation of the enantiomers. However, under our deposition conditions, with the substrate surface held at room temperature, the molecules are mobile and therefore an additional mixing entropy must be considered. In the disordered phase the racemate is favored by $R T \ln 2$, i.e., $1.73 \mathrm{~kJ} / \mathrm{mol}$ at $300 \mathrm{~K}$. MMC favor the enantiopure $\gamma$ phase over the $\lambda / \rho$ phase by only $1 \mathrm{~kJ} / \mathrm{mol}$. Consequently, the coverage needed for the $\gamma$ phase is not achieved at $300 \mathrm{~K}$. Deposition at lower temperatures does not lead to different results, since the mobility of the molecules becomes to low to undergo separation.

In the case of different local molecular adsorption geometries, lateral interactions vary substantially for a single compound with possible consequences for 
2D enantiomeric resolution. This has been shown for racemic tartaric acid (TA) on $\mathrm{Cu}(110)$ [19]. At different temperature and/or coverage, TA forms different lattice structures on the surface [20]. Depending whether one or both carboxylate groups react with the copper surface, bitartrate or monotartrate species will be present. For $(R, R)$-TA, bitartrate species have been identified only after thermal activation at lower coverage [20]. With increasing coverage, however, newly adsorbed TA molecules hydrogenate the doubly deprotonated bitartrate species to monotartrate again [20]. Besides this change in local adsorption geometry, an enantiomorphous $(90,12)^{*}$ bitartrate lattice undergoes a phase transition into a (4 0, 2 1) lattice. Interestingly, this lattice adopts the substrate symmetry, i.e., no chirality is expressed at the supramolecular level. With further exposure to enantiopure TA, a slightly denser (4 1, 2 5) structure is formed [20]. This structure breaks again the mirror symmetry of the underlying copper substrate, i.e., it is enantiomorphous. For racemic TA, on the other hand, a 2D conglomerate is observed only for the bitartrate species, where homochiral $(90,12)$ and $(90,-1$ 2) domains coexist on the surface [19]. The corresponding LEED pattern shows a superposition of both structures (Fig. 5). The racemate forms a $(40,21)$ monotartrate structure as well, but in contrast to the pure enantiomers, this is the monolayer saturation structure. This difference in achievable packing density for pure enantiomer and racemate is a strong indication that the two $(40,21)$ structures are different. For a conglomerate of coexisting (4 0, 2 1) mirror domains, further exposure to racemic TA should lead - equivalent to the $(90, \pm 1$ $2)$ bitartrate structures - to coexisting $(41, \pm 25)$ mirror domains. This difference is evidence for a heterochiral $(40,21)$ racemate lattice, not allowing additional inclusion of TA molecules. The lower thermal stability of the $(40,21)$ racemate structure with respect to the same structure of enantiopure TA supports this scenario [21]. Monotartrate decomposes upon heating into carbon dioxide, water, carbon and hydrogen on $\mathrm{Cu}(110)$. The stability of monotartrate is substantially enhanced due to the close-packed monolayer structure, not allowing interaction of upper parts of the molecule with the surface in the first place. Consequently, the $\mathrm{CO}_{2}$ TPD signal, reflecting the decomposition reaction, shifts to higher temperatures with increasing coverage (Fig. 5). Moreover, this reaction is catalyzed by free surfaces sites. Since the decomposition, in turn, creates active sites, the reaction is under autocatalytic control. Upon heating, this leads to complete decomposition in a very narrow temperature interval $(2 \mathrm{~K})$ once the reaction has started, because the gaseous decomposition products desorb instantaneously at decomposition temperature and a sharp pressure rise is observed. This is shown in Fig. 5 for $\mathrm{CO}_{2}$ as product. Interestingly, the decomposition temperature for the enantiopure $(40,21)$ structure exceeds the one for the same racemic structure by $8 \mathrm{~K}$. This higher stability is explained by a supramolecular chiral ensemble effect. The initial decomposition step requires a rearrangement of the upper part

* The numbers in parentheses stand for the $2 \times 2$ transformation matrix connecting adsorbate lattice with substrate surface lattice. 


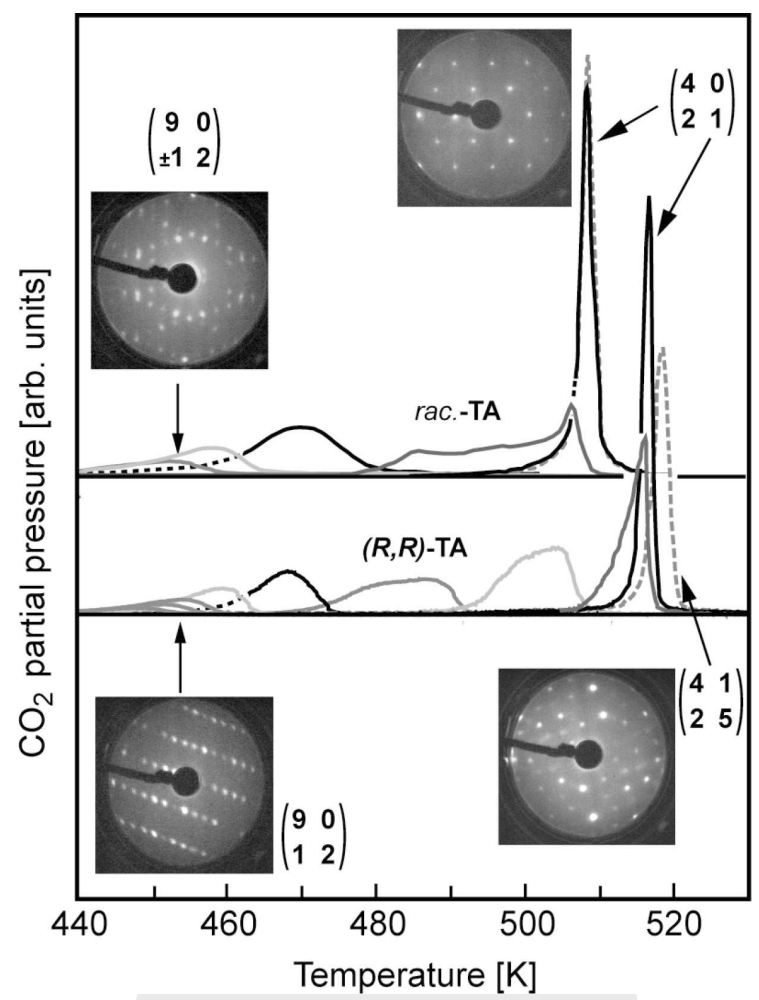

Fig. 5. LEED patterns and TPD curves for ( $R, R)$-TA (bottom) and racemic TA (top). At $405 \mathrm{~K}$, $(R, R)$-TA forms a $(90,12)$, a $(40,21)$ (not shown here) and a $(41,25)$ structure with increasing coverage. For the racemate a superposition of the mirror-related $\left(\begin{array}{llll}9 & 0,1 & 2\end{array}\right)$ and $(9$ $0,-12)$ domains as well as a $(40,21)$ structure are observed. The arrows point at the TPD traces for the respective lattice structures. While the thermal stability of the bitartrate structures for racemate and pure enantiomers is identical, it is lowered by $8 \mathrm{~K}$ for the racemate $(40,21)$ monotartrate structure compared to the $(R, R)-(40,21)$ monotartrate structure.

of the molecule in order to reach for the surface in a densely packed environment. The difference in handedness of adjacent molecules affects the extended H-bonding network of enantiopure and racemic lattices and has consequences for this initial "unhinging" process. Hence, the lower thermal stability is due to more heterogeneous bonding and leads again to the conclusion that the racemate forms a heterochiral $(40,21)$ monotartrate lattice. Consequently, the coexisting homochiral $(90, \pm 12)$ bitartrate domains show the same thermal stability as the $(R, R)$ $(90,12)$ bitartrate structure (Fig. 5).

Adsorption of achiral molecules onto achiral surfaces leads often to chiral adsorbate structures [11]. In principle, a single molecule may create a chiral motif on the surface, if belonging to the $\mathrm{C}_{\mathrm{nh}}, \mathrm{C}_{\mathrm{s}}$ or $\mathrm{C}_{\mathrm{i}}$ point group [13]. Either handedness 


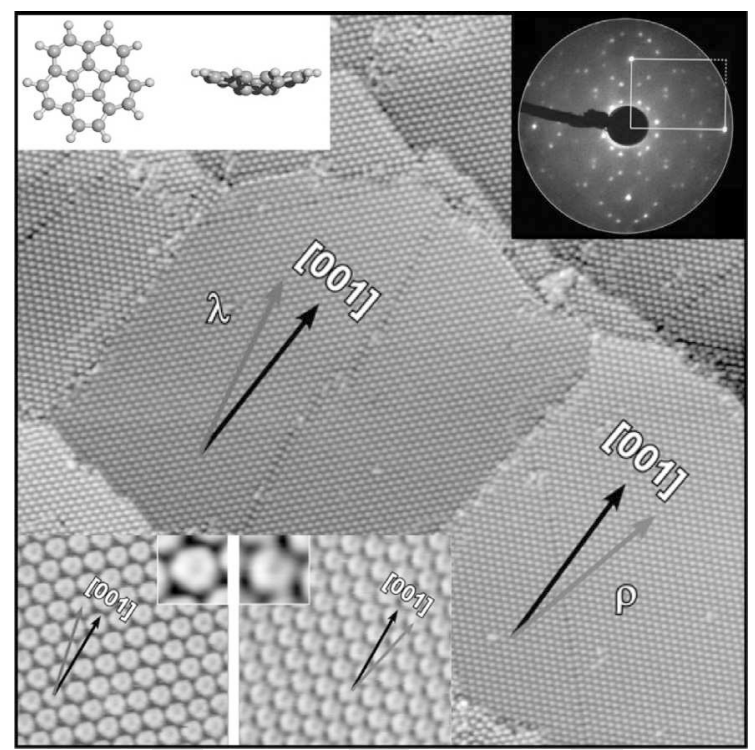

Fig. 6. LEED pattern and STM images of the corannulene monolayer on $\mathrm{Cu}(110)$. The symmetry mismatch between the substrate surface and the five-fold symmetric molecule leads to enantiomorphous packing arrangements. The LEED pattern is the superposition of the two mirror domains that are tilted to opposite directions. The highly resolved STM images (bottom insets) show that the molecule is oriented with the opening pointing away from the surface.

is then formed, depending on which enantiotopic side faces the surface. Breaking of mirror-symmetry is also induced by chiral distortion of the molecular frame, from different relative distances of the atoms relative to the surface, or by supramolecular and substrate lattice-induced chiral arrangements [11]. All adsorptioninduced chirality processes have in common that both enantiomers will be created as long as no further bias for single handedness is present. At a global level, the surface remains achiral, but at a local level, spontaneous symmetry breaking is a common phenomenon. As for intrinsically chiral molecules, the induced local chirality of adsorbed molecules is often transferred into the adlattice, which is then aligned in an oblique angle with respect to the substrate lattice. Again, these enantiomorphous structures are then easily observed via LEED.

Mirror domains might be just created, because the optimal packing arrangement breaks the symmetry of the underlying substrate lattice [11]. Corannulene, for example, forms spontaneously mirror domains on $\mathrm{Cu}(110)$ [22]. The bowlshaped fullerene fragment molecule has $\mathrm{C}_{5} \mathrm{v}$ symmetry. Since five-fold-symmetry cannot be maintained in a tiling array, a lattice belonging to the p1 plane group is formed. As manifestation of the enantiomorphism, the domains are tilted clockwise and counterclockwise in oblique angles with respect to the substrate lattice (Fig. 6). The LEED pattern therefore shows a superposition of both do- 
main lattices. In high-resolved STM images corannulene appears as doughnut, and comparison with results from semiempirical extended Hückel calculations strongly favors an adsorption geometry in which the bowl opening points away from the surface. X-ray photoelectron diffraction (XPD) measurements additionally revealed a small tilt of the $\mathrm{C}_{5}$-rotational axis away from the surface normal [22].

For $(R, R)$ - and $(S, S)$-bitartrate a zigzag distortion has been determined experimentally [23]. Density functional theory (DFT) calculations, however, also predict a chiral zigzag conformation after deprotonation of both carboxyl groups for achiral (R,S)-TA (meso-tartaric acid) and succinic acid (SU) on $\mathrm{Cu}(110)$ [24]. Experimental observations of long-range enantiomorphous patterns in connection with reasonable molecular structure considerations indeed suggest a chiral geometry for $(R, S)$-bitartrate and bisuccinate on $\mathrm{Cu}(110)[25,26]$. Because the probability of generating both enantiomorphous lattices is identical, two-dimensional conglomerates are formed, i.e., all molecules in a single domain of the adlattice have the same chirality. As in the case of racemic bitartrate and corannulene, both mirror structures are observed in the LEED pattern (Fig. 7, top), provided that the size of the probing electron beam is larger than the average domain size.

\subsection{Sergeants-and-soldiers}

As has been shown above, adsorption-induced chirality creates a bistable system with two distinct enantiomeric states. Additional chiral bias, however, can suppress one state if the barrier of interconversion is not too high. The coadsorption of chiral molecules into such racemic layers is an efficient way to induce further asymmetrization towards single handedness. A small amount of a chiral impurity can be sufficient for induction of homochirality on the entire surface! SU on $\mathrm{Cu}(110)$, for example, switches from one handedness to the other in its bisuccinate phase when the temperature is raised above $500 \mathrm{~K}$. Cooling down such layer after doping with one TA enantiomer completely suppresses the formation of one mirror domain and leads to global homochirality [27]. This process is illustrated in Fig. 7. Consequently, the opposite TA-enantiomer suppresses the opposite SU enantiomorph, which is easily detectable in LEED (Fig. 7). Only two percent of chiral dopant is necessary to install global homochirality. Smaller amounts of dopant lead to a lower intensity of the diffraction spots of the less favored enantiomorphous lattice. A similar effect - coined as sergeant-and-soldiers principle - has been observed for helical polymers where a small concentration of chiral side chains induced single helicity [28]. Since hydrogen bonds between the bisuccinate molecules as a means of transferring chirality can safely be excluded, one must consider a substrate mediated mechanism. That is, a chiral footprint in the surface acts as a chiral bias and suppresses opposite handedness in the adjacent adsorbate complex. This explains the amplification of the preferred handedness due to chiral doping: The TA sergeant has only one kind of a chiral footprint, forcing adjacent SU molecules into similar configuration. These, 


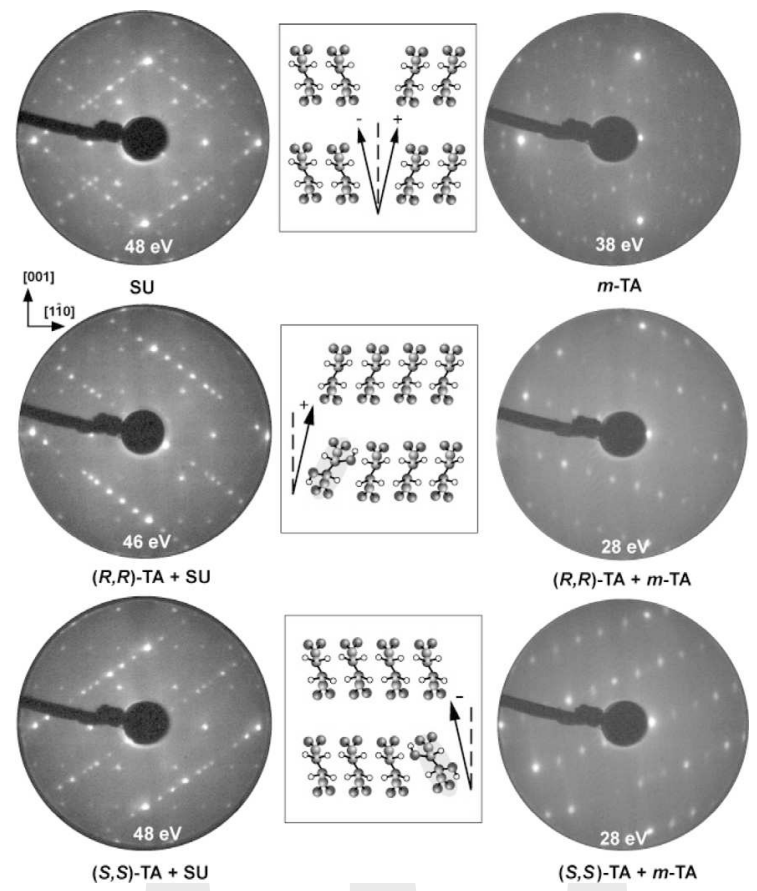

Fig. 7. Sergeant-and-soldiers principle at surfaces revealed by LEED: Prochiral SU and $(R, S)$ TA form conglomerates at the surface and both mirror domains are observed as superposition in the electron diffraction experiment (top row). Doping with chiral TA provides the chiral bias inducing homochirality in the entire $2 \mathrm{D}$ crystal. Electron beam energies and the $\mathrm{Cu}(110)$ lattice orientation are indicated.

in turn, force their SU neighbors into the same configuration for energetic reasons. The molecular frame of achiral $(R, S)$-TA on $\mathrm{Cu}(110)$ is forced into a zigzag distortion and becomes chiral as well. The sergeant-and-soldiers principle works therefore as well and homochirality induction is likewise observed after $(S, S)$ - or $(R, R)$-TA doping (Fig. 7, right column) [29].

\subsection{The majority rules!}

In contrast to the chiral doping mechanism, small ee may also induce lattice homochirality [30]. As mentioned above, the heterochiral pairs of [7] $\mathrm{H}$ on $\mathrm{Cu}(111)$ may exist in two enantiomorphous states due to two possible alignments on the $\mathrm{Cu}(111)$ surface. The chiral bias from a small ee is sufficient in order to suppress formation of one unfavorable mirror domain and induces homochiral lattice order [15]. Because of strong steric constraints, any ee is expelled from 
the racemic enantiomorphous domains during crystallization. From the domain edges, however, the excess molecules have an influence on the relative alignment of the heterochiral pairs at the domain edge. $(M)$-[7] $\mathrm{H}$ excess favors formation of $\lambda$ domain pairs and $(P)-[7] \mathrm{H}$ excess favors the $\rho$ domain-pair-alignment. This chiral bias is then amplified by the cooperative interaction among heterochiral pairs, strongly favoring their equal alignment. Like helix reversals in a polymer chain, opposite alignment would create energetically unfavorable mirror domain boundaries. Even for the pure racemate, these boundaries are rarely observed on single $\mathrm{Cu}(111)$ terraces. Hence, without any chiral bias, spontaneous symmetry breaking is observed on this length scale, but the probability for formation of $\lambda$ and $\rho$ domain decorated terraces is equal. At ee $= \pm 0.08$ the entire surface although still close to racemic content - is already driven into a homochiral arrangement during 2D crystallization and only one of either $\lambda$ or $\rho$ domains is observed (Fig. 8). In contrast to the SU/TA-doped and $(R, S)$-TA/TA doped systems, where the molecular frame of the molecule is switched to its mirror configuration, interconversion between both structures requires only a change in relative position of both enantiomers of a heterochiral pair. The TA-doped systems are truly homochiral, i.e., all molecules have the same handedness. The eebias-amplified $[7] \mathrm{H} / \mathrm{Cu}(111)$ system, however, is still of heterochiral composition, but possesses a lattice homochirality. In order to observe this ee-effect, lateral resolution of the enantiomers is not allowed.

\section{Conclusions and outlook}

Long-range chiral motifs are formed after adsorption of chiral molecules or due to spontaneous symmetry breaking of prochiral molecules. Additional chiral bias due to chiral impurities (dopant) or small enantiomeric excess leads via cooperative amplification to homochirality in the 2D molecular lattice. Chirality is a wonderful approach in order to better understand complex molecular interactions. Many cooperative phenomena ruling supramolecular processes are still waiting to be understood and to be described at a molecular level. From the riddle of the transfer of molecular structure into the shape of (enantiomorphous) crystals to the influence of small impurities deciding the outcome of macroscopic shape, 2D model systems help to understand these complex mechanisms. Beyond that, well-characterized 2D systems are an excellent starting point in studying the structural transfer mechanisms into the 3D world. Chiral surface science is still in its infancy, and most of the information has been gathered during the last few years only. With the further development of experimental and theoretical tools, in particular density functional theory (DFT), much new insight on intermolecular interactions will be achieved in the near future. 

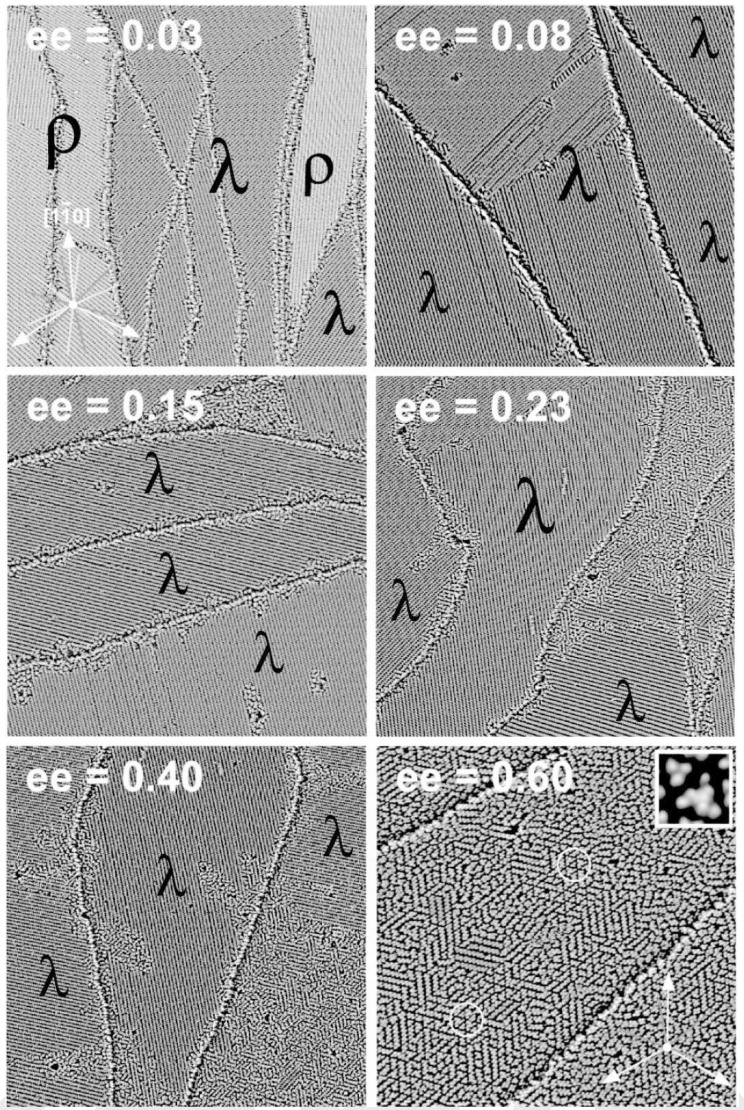

Fig. 8. STM images $(200 \mathrm{~nm} \times 200 \mathrm{~nm})$ of [7]H on $\mathrm{Cu}(111)$ with increasing ee. At ee $=0.08$ only a single mirror domain type $(\lambda)$ can exist on the surface. The excess is expelled from the heterochiral domain and with increasing ee, the excess area increases accordingly. The circles highlight pinwheel motifs observed otherwise for the pure enantiomers (inset).

\section{Acknowledgement}

Support by the Schweizerischer Nationalfonds is gratefully acknowledged. I thank all members of Empa's Molecular Surface Science Group, namely, B. Behzadi, M. Böhringer, A. Cossy, R. Fasel, Y. Kuster, M. Parschau, S. Romer, who contributed to this work over the last ten years as well as U. Ellerbeck and Jay Siegel for heptahelicene and corannulene synthesis. 


\section{References}

1. L. Pasteur, Ann. Chim. Phys. 24 (1848) 442.

2. J. D. Dunitz, Chem. Comm. (2003) 545.

3. J. Jacques, A. Collet, S. H. Wilen, Enantiomers, Racmates and Resolutions. Krieger Publishing Company, Malabar, Florida (1994).

4. C. P. Brock, W. B. Schweitzer, J. D. Dunitz, J. Am. Chem. Soc. 113 (1991) 9811.

5. J. S. Siegel, Nature 409 (2001) 777.

6. K.-H. Ernst, D. Schlatterbeck, K. Christmann, Phys. Chem. Chem. Phys. 1 (1999) 4105 .

7. A. Sudharkar, T. J. Katz, Tetrahedron Lett. 27 (1986) 2231.

8. K.-H. Ernst, Y. Kuster, R. Fasel, M. Müller, U. Ellerbeck, Chirality 13 (2001) 675.

9. T. Bürgi, A. Urakawa, B. Behzadi, K.-H. Ernst, A. Baiker, New J. Chem. 28 (2004) 332.

10. R. Fasel, A. Cossy, K.-H. Ernst, F. Baumberger, T. Greber, J. Osterwalder, J. Chem. Phys. 115 (2001) 1020.

11. K.-H. Ernst, Top. Curr. Chem. 265 (2006) 209.

12. R. Fasel, M. Parschau, K.-H. Ernst, Angew. Chem. Int. Ed. 42 (2003) 5178; Angew. Chem. 115 (2003) 5336.

13. S. M. Barlow, R. Raval, Surf. Sci. Rep. 50 (2003) 201.

14. W. Stocker, M. Schumacher, S. Graff, A. Thierry, J. C. Wittmann, B. Lotz, Macromolecules 31 (1998) 807.

15. R. Fasel, M. Parschau, K.-H. Ernst, Nature 439 (2006) 449.

16. M. Parschau, R. Fasel, K.-H. Ernst, Cryst. Growth \& Des. 8 (2008) 1890.

17. K.-H. Ernst, M. Neuber, M. Grunze, U. Ellerbeck, J. Am. Chem. Soc. 123 (2001) 493.

18. K.-H. Ernst, Y. Kuster, R. Fasel, C. F. McFadden, U. Ellerbeck, Surf. Sci. 530 (2003) 195.

19. S. Romer, B. Behzadi, R. Fasel, K.-H. Ernst, Chem. Eur. J. 11 (2005) 4149.

20. M. Ortega Lorenzo, S. Haq, T. Bertrams, P. Murray, R. Raval, C. J. Baddeley, J. Phys. Chem. B 103 (1999) 10661.

21. B. Behzadi, S. Romer, R. Fasel, K.-H. Ernst, J. Am. Chem. Soc. 126 (2004) 9176.

22. M. Parschau, R. Fasel, K.-H. Ernst, O. Gröning, L. Brandenberger, R. Schillinger, T. Greber, A. Seitsonen, Y.-T. Wu, J. S. Siegel, Angew. Chem. Int. Ed. 46 (2007) 8258; Angew. Chem. 119 (2007) 8406.

23. R. Fasel, J. Wider, C. Quitmann, K.-H. Ernst, T. Greber, Angew. Chem. Int. Ed. 43 (2004) 2853.

24. L. A. M. M. Barbosa, P. Sautet, J. Am. Chem. Soc. 123 (2001) 6639.

25. V. Humblot, M. Ortega Lorenzo, C. J. Baddeley, S. Haq, R. Raval, J. Am. Chem. Soc. 126 (2004) 6460.

26. M. Parschau, B. Behzadi, S. Romer, K.-H. Ernst, Surf. Interface Anal. 38 (2006) 1607

27. M. Parschau, S. Romer, K.-H. Ernst, J. Am. Chem. Soc. 126 (2004) 15398.

28. M. M. Green, M. P. Reidy, R. J. Johnson, G. Darling, D. J. O’Leary, G. Wilson, J. Am. Chem. Soc. 111 (1989) 6454.

29. M. Parschau, T. Kampen, K.-H. Ernst, Chem. Phys. Lett. 407 (2005) 433.

30. K.-H. Ernst, Curr. Opin. Coll. Interf. Sci. 13 (2008) 54. 


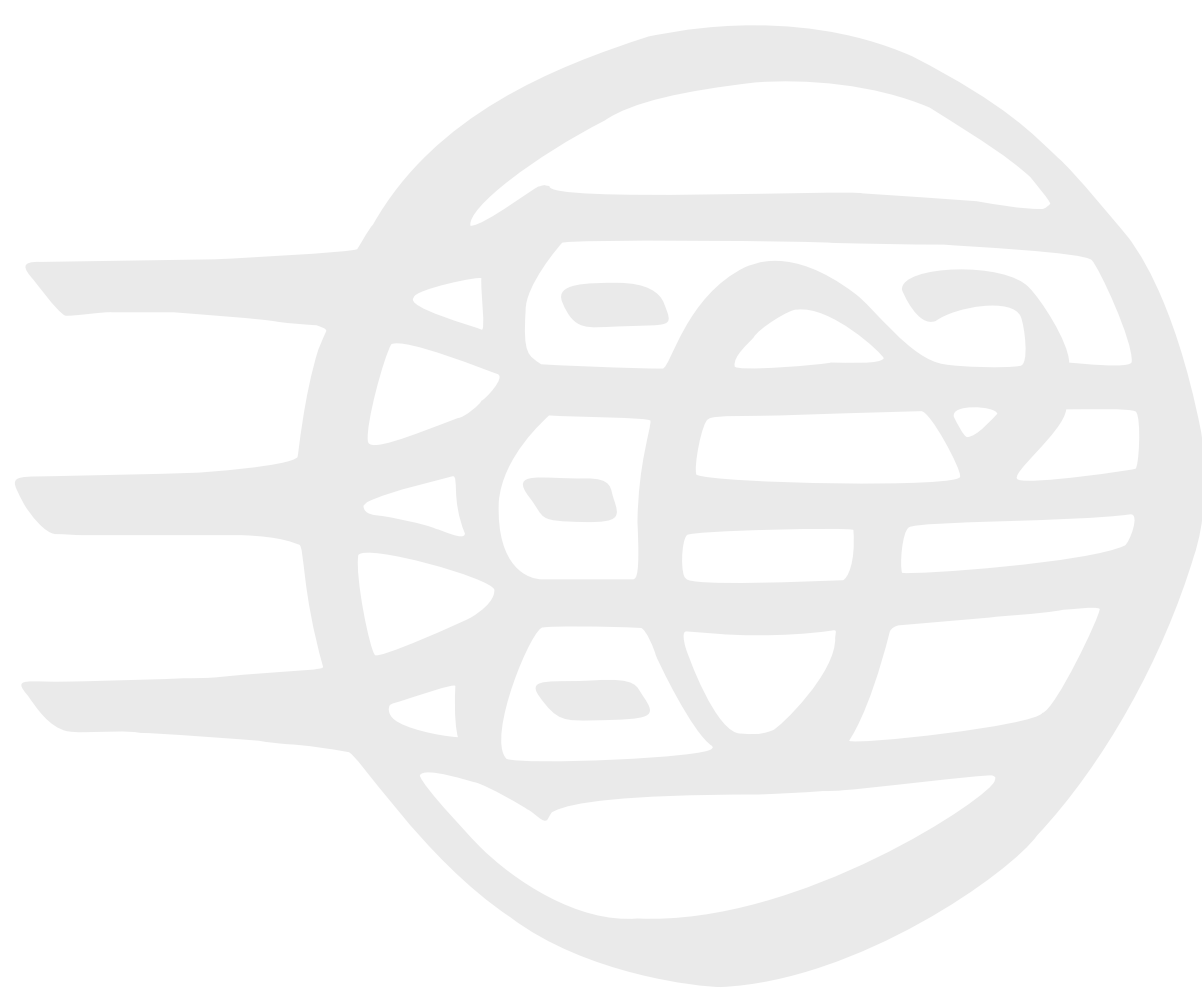

